\title{
Combined dynamic spectral imaging and routine colposcopy strategy for the diagnosis of pre-cancerous cervical lesions
}

\author{
DAN LIU and WANLIANG HU
}

\author{
Department of Medicine, Jinggangshan University, Ji'an, Jiangxi 343009, P.R. China
}

Received April 28, 2018; Accepted December 21, 2018

DOI: $10.3892 /$ etm.2019.7719

\begin{abstract}
The present study aimed to investigate the sensitivity and specificity of combined dynamic spectral imaging (DySI) and routine colposcopy in diagnosing pre-cancerous lesions in subjects with abnormal cytological results. The retrospective study included 146 patients diagnosed with cervical lesions and atypical squamous cells of undetermined significance according to a colposcopy examination. Data from colposcopy clinics were used to evaluate performance of DySI in different histological types. The present study evaluated the clinical performance of two different referral strategies (single diagnosis or combined diagnosis) in combination with a smear test. The sensitivity of DySI and routine colposcopy for detecting severe pre-cancerous cervical disease in patients referred with a borderline histology or mild dyskaryosis was $69 \%[95 \%$ confidence interval (CI): 56-82\%] and 43\% (95\% CI: 28-57\%), respectively $(\mathrm{P}=0.01)$. However, for the HSIL group, the sensitivity of DySI and routine colposcopy was 64 and $61 \%$, respectively. In the HSIL group, the sensitivity was as high as $85 \%$ when the DySI method was combined with routine colposcopy. When the conditions of new screening strategies were applied to remove those patients with a negligible risk, DySI had a higher sensitivity to detect severe pre-cancerous cervical disease than conventional colposcopy. In conclusion, the combined DySI and routine colposcopy strategy had a higher diagnostic sensitivity in subjects with low or high abnormalities on cytological examination, compared with either method separately. The present study suggests that the baseline colposcopy sensitivity may be enhanced with the adjunctive use of DySI, irrespective of the cytology result for referral.
\end{abstract}

Correspondence to: Professor Wanliang Hu, Department of Medicine, Jinggangshan University, 28 Xueyuan Road, Ji'an, Jiangxi 343009, P.R. China

E-mail: jxjaliudan@163.com

Key words: dynamic spectral imaging, cervical intraepithelial neoplasia, sensitivity and specificity, high-risk human papilloma virus

\section{Introduction}

Cervical carcinoma is the second most common malignant tumor type in females, accounting for 527,600 newly diagnosed cases and 265,700 deaths worldwide in 2012 (1). The neoplasm is most frequently caused by persistent infection with high-risk human papillomavirus (HPV), leading to slow and progressive infiltration $(2,3)$. Usually, the process of cervical carcinogenesis takes 10 years or longer $(2,3)$. Regular and frequent cervical carcinoma screening and early diagnostic approaches have become the most critical tools for preventing the progression of pre-cancerous lesions to cervical carcinoma (4). Colposcopy is a visual technique that is extensively used to collect cervical tissue samples and identify cervical lesions. However, the application of colposcopy is limited by the skills and experience of clinical staff (5). Furthermore, the selection of the biopsy site is always subjective and affects the determination of the histological type based on visual changes (6). Due to the above reasons, the accuracy rate of the determination of cell morphological changes and mild nuclear abnormalities is limited. At present, the predictive rate for severe pre-cancerous lesions is $<55 \%$ in the clinic (7). Aberrant cell morphology and mild nuclear abnormalities always induce atypical squamous cells of undetermined significance (ASCUS)/atypical squamous cells-cannot exclude high-grade squamous intraepithelial lesion (ASC-H)/low-grade squamous intraepithelial lesion (LSIL). Furthermore, high-grade squamous intraepithelial lesions (HSIL) are considered severe pre-cancerous lesions (8).

The dynamic spectral imaging (DySI) colposcope is a digital-video colposcope integrating standard colposcopy with adjunctive DySI cervical mapping (9). DySI has a relatively higher sensitivity for detecting severe pre-cancerous lesions [defined as lesions covering $>2 / 3$ of the total uterine neck and cervical intraepithelial neoplasia 2+ (CIN2+)] (10). Soutter et al (11) reported that the diagnostic efficacy for CIN2+ lesions by DySI is significantly higher compared with that of traditional colposcopy in patients with cytological abnormalities. In 2013, the American Cancer Society updated their screening guidelines for the early detection of cervical carcinoma and pre-cancerous lesions (12), to recommend the cytological technique as the discriminant diagnostic tool for high-risk (hr)HPV-positive patients according to the World Health Organization Guidelines (13). At present, the determination of hrHPV is considered as an alternative method after 
the cytological examination, while the colposcopy examination is performed directly for those patients with severe cytological results (14).

In the present study, the diagnostic efficacy of DySI, traditional colposcopy and their combination was first evaluated. Subsequently, two different screening methods of traditional colposcopy, namely hrHPV examination as the pre-screening method and cytological examination combined with determination of the hrHPV, were compared. The results revealed that the combined application of the DySI technique and traditional colposcopy exhibited a higher sensitivity compared with that of traditional colposcopy alone for the detection of pre-cancerous cervical lesions in subjects with an abnormal cytological result.

\section{Materials and methods}

Patients. The present retrospective study included 146 patients diagnosed with cervical lesions and ASCUS on colposcopy examination. The inclusion criteria were an age of $>18$ years, and an abnormal screening test result [referral from cervical screening to the colposcopy unit due to an abnormal cervical cytological result]. Patients fulfilling the following criteria were excluded: i) Known pregnancy, ii) human immunodeficiency virus infection, iii) acquired immune deficiency syndrome, iv) hysterectomy and v) radiation treatment or chemotherapy.

DySI examination. The DySI system used in the present study was a DySIS v2.1 purchased from Forth Photonics Ltd. (Livingston, UK). This technique allowed for visualization of the cervix during a colposcopic examination and measurement or mapping the dynamics of the acetowhitening effects for each point of the cervix, to finally provide a per-point analysis of the acetowhitening effects.

Upon addition of 3\% acetic acid, the evaluation of acetowhitening effects by DySI commenced automatically. Although the acquisition period continued for $\sim 3 \mathrm{~min}$, the acetowhitening effects persisted for longer (15). When the examination was completed, the DySI information was concisely illustrated with a color-coded map. The color-coded map was overlaid with the color images for the vertical tissues to facilitate the identification of the location of cervical lesions and determine the severity. The DySI procedure was performed according to the manufacturer's protocols.

hrHPV screening. The patients were instructed to refrain from sexual activity and washing of the vagina, and no drugs were applied and no other surgeries of the cervix and vagina were performed for 3 days prior to the HPV screening. The cervix was exposed by with a speculum and a HPV sampler was used to obtain the specimens. The specimens were preserved in PBS and ready to be detected in the laboratory. hrHPV was assessed in the cervical specimens by employing the GP5+/6+ polymerase chain reaction enzyme immunoassay according to the protocols of previous studies $(16,17)$.

Evaluation of clinical presentation by two different strategies. The patients of the present study were divided into two groups that were subjected to different evaluation strategies prior to colposcopy and/or DySI (18). First, hrHPV examination was used as the pre-screening experiment. If the hrHPV test was positive, the patients were separated and then subjected to the diagnostic procedures. Meanwhile, the hrHPV negative patients were also subjected to the diagnostic procedures for consistency. Furthermore, hrHPV examination was used as the criterion for classification examination. If patients had a critical cell morphology or mild nuclear abnormalities and an hrHPV-positive status, the patients were separated and then subjected to the diagnostic procedures. The hrHPV negative patients were also subjected to the diagnostic procedures. If the patients' cytological results indicated a morphology of highly diseased cells, the patients were selected directly and subjected to the diagnostic procedures immediately.

Trial grouping. According to the presence of abnormal nuclear morphology and the degree of pre-cancerous lesions, the patients were divided into an ASCUS/ASC-H/LSIL group $(n=93)$, HSIL group $(n=53)$ and HSIL/hrHPV-negative group $(n=13)$. Furthermore, all three groups were subdivided into a routine colposcopy group, DySI group and combined diagnosis by DySI and routine colposcopy group. In addition, the patients were divided into two groups that were subjected to different evaluation strategies to examine the clinical presentation prior to colposcopy. For the Method 1 group $(n=110)$, the hrHPV examination was used as the pre-screening experiment, and for the Method 2 group $(n=124)$, hrHPV examination was used as a criterion for classification examination (cytology analysis). Each of the two groups was sub-divided into a routine colposcopy group, a DySI group and a combined diagnosis by DySI and routine colposcopy group.

Statistical analysis. All of the data were analyzed by using SPSS software 19.0 (IBM Corp., Armonk, NY, USA). Quantitative data are expressed as the mean \pm standard deviation. Categorical variables, including sensitivity and specificity (expressed as percentages, \%), were analyzed by using a Chi-square test. Receiver operating characteristic curves were employed to evaluate the sensitivity and specificity of the routine colposcopy method, DySI and the combined diagnostic method, according to previously published studies $(19,20)$. All of the data were obtained from at least six independent tests or experiments. $\mathrm{P}<0.05$ was considered to indicate a statistically significant difference.

\section{Results}

Histological results. The present study included 146 patients with pre-cancerous lesions according to the patients' cytological results. The histological samples were taken from the cervix of the uterus using the colposcope prior to the analysis of the diagnostic sensitivity and specificity according to a previously published study (21). In the current study, the gold standard for the cancer diagnosis was the histological analysis. Histological analysis revealed that the patients either exhibited focal advanced squamous intraepithelial lesions (CIN2, CIN3; 66 patients; Fig. 1A), low-grade squamous intraepithelial lesions (CIN1; 41 patients; Fig. 1B) or low-grade intraepithelial lesions (CIN0; 37 patients; Fig. 1C). There were also two patients with adenocarcinoma of uterine cervix. Therefore, all of the patients were included in the subsequent examinations. 

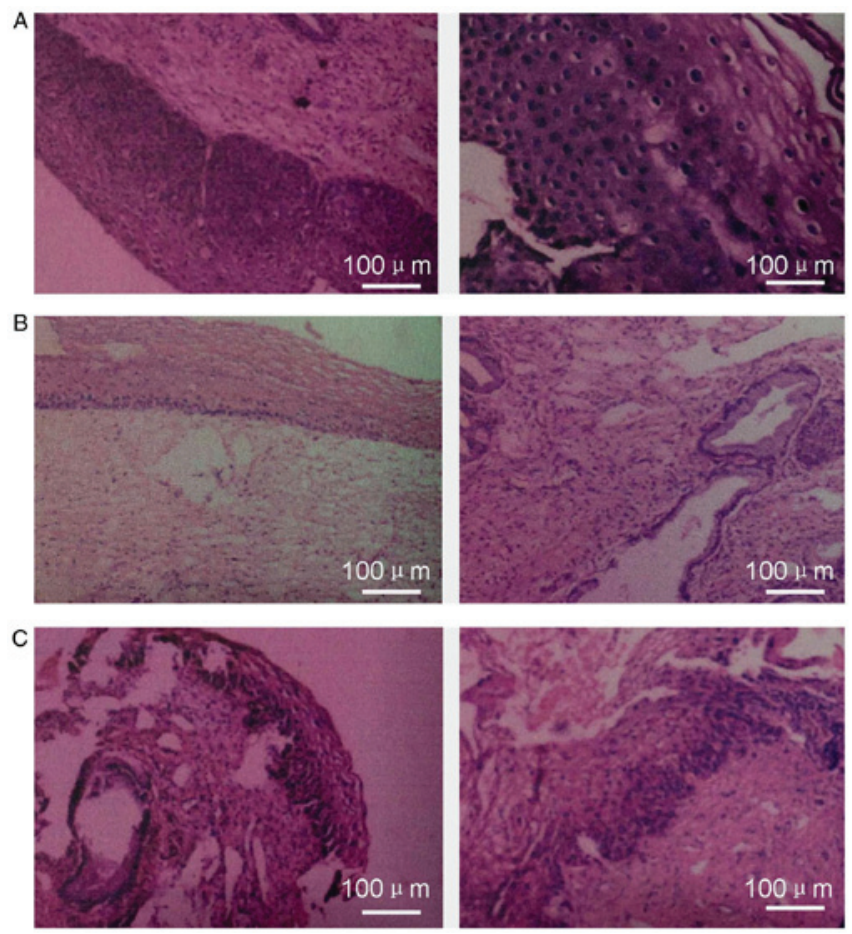

Figure 1. Representative histological images. (A) Advanced squamous intraepithelial lesion (CIN2, CIN3). (B) Low-grade squamous intraepithelial lesion (CIN1). (C) Low-grade intraepithelial lesion (scale bar, $100 \mu \mathrm{m}$ ). CIN, cervical intraepithelial neoplasia.

Characteristics of the patients. A total of 93 patients $(63.7 \%)$ were indicated to have low cytological abnormalities and 53 patients $(36.3 \%)$ had high cytological abnormalities (Table I). Furthermore, the lesions of 110 patients $(75.3 \%)$ were hrHPV-positive and those of 33 patients (22.6\%) were hrHPV-negative, while no results were obtained for 3 patients (3.1\%). Regarding the CIN grades, 37 patients were CINO, 41 were CIN1, 14 were CIN2 and 52 were CIN3 grade; furthermore, 2 patients were diagnosed with adenocarcinoma of the cervix (Table I). Two biopsy specimens were collected from each patient for the subsequent tests.

Combination of routine colposcopy and DySI enhances the diagnostic sensitivity for pre-cancerous lesions in subjects with abnormal cytological results. In patients classified as ASCUS/ASC-H/LSIL, the diagnostic sensitivity in the DySI subgroup [69\%; 95\% confidence interval (CI), 56-82\%] was significantly higher compared with that in the routine colposcopy subgroup (43\%; 95\% CI, 28-57\%; $\mathrm{P}=0.01$; Table II). When the DySI method was combined with routine colposcopy, the diagnostic sensitivity was increased to $77 \%$ (95\% CI, 65-88\%), which was significantly higher compared with that of routine colposcopy alone and DySI alone, respectively (all $\mathrm{P}<0.05$; Table II). Furthermore, in the HSIL group, combined diagnosis by DySI and routine colposcopy also demonstrated a higher diagnostic sensitivity compared with that of in the routine colposcopy alone or DySI alone group $(\mathrm{P}<0.05$; Table II). However, there were no significant differences in the diagnostic sensitivity among the three subgroups for the HSIL and hrHPV-negative group $(\mathrm{P}<0.05$; Table II).
Table I. Clinicopathological characteristics of the subjects $(n=146)$.

\begin{tabular}{lc}
\hline Item & Value \\
\hline Age (years) & \\
Mean (range) & $37.0(18.7-62.6)$ \\
Median & 35.4 \\
Cytological detection & \\
ASCUS/ASC-H/LSIL & $93(63.7)$ \\
HSIL & $53(36.3)$ \\
hrHPV detection & \\
Positive & $110(75.3)$ \\
Negative & $33(22.6)$ \\
Ineffective detection & $3(2.1)$ \\
Grade of tumorigenicity & \\
CIN0 & $37(25.3)$ \\
CIN1 & $41(28.1)$ \\
CIN2 & $14(9.5)$ \\
CIN3 & $52(35.6)$ \\
Adenocarcinoma of uterine cervix & $2(1.4)$ \\
\end{tabular}

Values are expressed as $\mathrm{n}(\%)$ unless otherwise specified. CIN, cervical intraepithelial neoplasia; hrHPV, high-risk human papillomavirus; ASCUS, atypical squamous cells of undetermined significance; ASC-H, atypical squamous cells-cannot exclude high-grade squamous intraepithelial lesion; LSIL, low-grade squamous intraepithelial lesion; HSIL, high-grade squamous intraepithelial lesion.

Cytological examination according to Method 1. In Method 1 (a pre-screening strategy), determination of the hrHPV status was used as the pre-screening tool, and cytological examination was then employed to make a definite diagnosis for patients with hrHPV-positive samples. In the present study, 110 out of 146 patients $(75.3 \%)$ were hrHPV-positive and subjected to a cervical smear test. The results indicated that 71 patients $(64.5 \%)$ had low cytological abnormalities and 39 patients (35.5\%) had high cytological abnormalities (Table III).

Cytological examination according to Method 2. In Method 2 (a pre-screening strategy), the patients with low cytological changes and hrHPV-negative status were excluded from further analysis. Therefore, all of the patients with high cytological changes and those with low cytological abnormalities and hrHPV-positive status were subjected to examination by colposcopy and/or DySI. The results indicated that 93 patients (76.4\%) exhibited low cytological abnormalities and were hrHPV-positive, and 53 patients exhibited high cytological abnormalities (Table III). Therefore, 124 patients ( $84.9 \%$ of all patients) were included in Method 2; these patients included 71 hrHPV-positive patients and 53 patients who illustrated higher cytological abnormalities.

DySI enhances the diagnostic sensitivity for subjects with $\geq$ CIN2+ pre-screened with Method 1 or 2 . If Method 1 was used for pre-screening, the diagnostic sensitivity for detecting (pre-)cancerous lesions in subjects with CIN2+ in the DySI 
Table II. Sensitivity and specificity of routine colposcopy, DySI and their combination in the diagnosis of intraepithelial cervical lesions of different histological types (threshold, CIN2+) as pre-cancerous lesions.

\begin{tabular}{|c|c|c|c|c|c|}
\hline Group & $\mathrm{N}$ & Sensitivity (95\% CI), \% & P-value & Specificity (95\% CI), \% & P-value \\
\hline ASCUS/ASC-H/LSIL & 93 & & & & \\
\hline Routine colposcopy & & $43(28-57)$ & & $88(81-95)$ & \\
\hline DySI & & $69(56-82)$ & 0.010 & $78(68-88)$ & 0.210 \\
\hline Combined diagnosis & & $77(65-88)$ & 0.001 & $70(59-80)$ & 0.001 \\
\hline HSIL & 53 & & & & \\
\hline Routine colposcopy & & $61(48-74)$ & & $55(35-75)$ & \\
\hline DySI & & $64(51-76)$ & 1.000 & $63(42-83)$ & 0.690 \\
\hline Combined diagnosis & & $85(75-95)$ & 0.001 & $25(25-65)$ & 0.500 \\
\hline HSIL and hrHPV negative & 13 & & & & \\
\hline Routine colposcopy & & $55(22-87)$ & & $46(16-75)$ & \\
\hline DySI & & $34(4-65)$ & 0.690 & $65(36-93)$ & 0.630 \\
\hline Combined diagnosis & & $78(52-100)$ & 0.500 & $36(7-66)$ & 1.000 \\
\hline
\end{tabular}

Table III. Influence of different referral strategies on the sensitivity and specificity of routine colposcopy, DySI and their combination in the diagnosis of intraepithelial cervical lesions (threshold, CIN2+) as pre-cancerous lesions.

\begin{tabular}{lcccc}
\hline Method & $\mathrm{N}$ & $\begin{array}{c}\text { Advanced lesions } \\
\text { under colposcopy, }(\%)\end{array}$ & $\begin{array}{c}\text { Sensitivity } \\
(95 \% \text { CI }), \%\end{array}$ & $\begin{array}{c}\text { Specificity } \\
(95 \% \text { CI }), \%\end{array}$ \\
\hline 1 & 110 & $59(53.3)$ & & $83(74-91)$ \\
P-value
\end{tabular}

aP-values refer to comparison with routine colposcopy group. CI, confidence interval; DySI, dynamic spectral imaging; CIN, cervical intraepithelial neoplasia; CIN, cervical intraepithelial neoplasia.

group (67\%; 95\% CI, 57-77\%) was significantly higher compared with that in the routine colposcopy group $(52 \%$; 95\% CI, 42-63\%; P=0.04; Table III). Furthermore, compared with that in the groups using either diagnostic method alone, the diagnostic sensitivity achieved by combination of routine colposcopy with DySI was significantly increased to $82 \%$ (95\% CI, 73-90\%; P<0.05; Table III). If Method 2 was used for pre-screening, no significant differences in the diagnostic sensitivity were obtained between the DySI group (64\%; $95 \%$ CI, 54-73\%) and the routine colposcopy group (53\%; 95\% CI, 43-63\%; P<0.05; Table III). However, the diagnostic sensitivity in the combined diagnosis group (81\%; 95\% CI, 74-89\%) was significantly higher compared with that in the groups using either diagnostic method alone ( $\mathrm{P}=0.001$; Table III).

\section{Discussion}

Although cervical screening strategies have proven to be successful in preventing cervical carcinoma, the diagnostic accuracy also requires to be improved. Therefore, the diagnostic accuracy of routine colposcopy combined with DySI was investigated in the present study. It was revealed that DySI exhibited a higher sensitivity, which is consistent with the result of a previously published study (10). However, it is uncommon that hrHPV-negative patients exhibit high cytological changes; this may occur as the sequelae of un-associated risk factors.

The finding of low cytological abnormalities accounts for the overwhelming majority of abnormal cytological morphologies in screening processes (22), and represents 
the patients who were remained undiagnosed following the colposcopy. Due to a few factors, such as difficulties in diagnosis and economic burden of patients, international research to improve the screening process and diagnostic methods for cervical carcinoma has been launched. In fact, the implementation of the hrHPV examinations led to subjects undergoing colposcopy screening in the clinic more frequently, a certain amount of which may be unnecessary. Furthermore, potential mistakes, including the relatively lower specificity of combined diagnostic methods, or misdiagnosis (false-positive diagnoses or identifying a low number of cytological abnormalities) may cause unnecessary anxiety of patients, along with increased national healthcare costs. However, it is important to perform preliminary screenings prior to examination for cervical carcinoma using combined diagnostic methods with enhanced sensitivity, while those patients not fulfilling certain criteria may be excluded from further examinations. In the present study, the data were analyzed following DySI examination, and the results suggested that DySI combined with routine colposcopy is able to significantly improve the accuracy and sensitivity of cervical carcinoma diagnosis by first subjecting the patients to hrHPV examination and/or cytological examination $(10,23)$.

In recent years, novel methods for diagnosing pre-cancerous lesions have been proposed. Ni et al (24), used endoscopic diagnosis for diagnosing pre-cancerous laryngeal lesions, which is also a traditional method for examining laryngeal cancer lesions. DeNardis et al (25), applied adjunctive dynamic spectral imaging to detect pre-cancerous cervical lesions. However, the secondary sensitivity and specificity of the adjunctive dynamic spectral imaging on its own are relatively low. Furthermore, in the present study, the strategy of combining the DySI and routine colposcopy was applied for the first time, to the best of our knowledge, which is also different from the method described by DeNardis et al (25). In addition, three-dimensional power Doppler ultrasound was previously utilized to diagnose pre-cancerous lesions, but the sensitivity of this method is lower (26) compared to the present study. It is therefore indicated that the combination of DySI and routine colposcopy is a promising strategy for diagnosing pre-cancerous cervical lesions.

The present study had two possible limitations. First, when the different screening methods and the referral method were retrospectively simulated, certain deviations in the data might occur. There is controversy regarding the correlation between the characteristics of CIN2 and the clinical histological type, however, CIN2 is also the threshold for excision treatment $(27,28)$. Furthermore, the number of biopsies from the same patient were relatively small in the present study. Increasing the amount of biopsies may increase the sensitivity of the colposcopy examination (29). However, in the present study, a mean number of specimens of 2 (ranging from 1 to 4 ) was selected, which is consistent with the normal amount of biopsy specimens in the clinic. Therefore, it is indicated that any potential effects of the amounts of selected tissue biopsy specimens may be neglected.

In conclusion, the present study proved that the DySI strategy has a higher diagnostic sensitivity compared with that of routine colposcopy. Furthermore, adjuvant DySI improves the accuracy of colposcopy examination in different setups. In conclusion, combined DySI and routine colposcopy exhibited an increased diagnostic sensitivity for the detection of pre-cancerous lesions in patients with low or high cytological abnormalities, compared with either method on its own.

\section{Acknowledgements}

Not applicable.

\section{Funding}

The present study was granted by the Science and Technology Project of Jiangxi Education Department in 2017 (grant no. GJJ170629).

\section{Availability of data and materials}

All data generated or analyzed during the present study are included in this published article.

\section{Authors' contributions}

WLH designed the current study and provided the fund. DL and WLH performed the experiments, collected the patients' data, wrote the manuscript, and read and approved the final manuscript.

\section{Ethical approval and consent to participate}

The present study was approved by the Ethics Committee of Jinggangshan University (Ji'an, China). All of the patients provided their written informed consents to participate in the present study.

\section{Patient consent for publication}

Not applicable.

\section{Competing interests}

The authors declare that they have no competing financial or commercial interests regarding the present study.

\section{References}

1. Torre LA, Bray F, Siegel RL, Ferlay J,Lortet-Tieulent J and Jemal A: Global cancer statistics, 2012. CA Cancer J Clin 65: 87-108, 2015.

2. Luo S, Li N, Yu S, Chen L, Liu C and Rong J: MicroRNA-92a promotes cell viability and invasion in cervical cancer via directly targeting Dickkopf-related protein 3. Exp Ther Med 14: 1227-1234, 2017.

3. Li B, Shi H, Wang F, Hong D, Lv W, Xie X and Cheng X: Expression of E-, P- and N-cadherin and its clinical significance in cervical squamous cell carcinoma and precancerous lesions. PLoS One 11: e0155910, 2016.

4. Wang JL, Yang YZ, Dong WW, Sun J, Tao HT, Li RX and Hu Y: Application of human papillomavirus in screening for cervical cancer and precancerous lesions. Asian Pac J Cancer Prev 14: 2979-2982, 2013

5. Ma L, Cong X, Shi M, Wang XH, Liu HY and Bian ML: Distribution of human papillomavirus genotypes in cervical lesions. Exp Ther Med 13: 535-541, 2017. 
6. Wentzensen N, Zuna RE, Sherman ME, Gold MA, Schiffman M, Dunn ST, Jeronimo J, Zhang R, Walker J and Wang SS: Accuracy of cervical specimens obtained for biomarker studies in women with CIN3. Gynecol Oncol 115: 493-496, 2009.

7. Ferris DG, Litaker MS and ALTS Group: Prediction of cervical histologic results using an abbreviated reid colposcopic index during ALTS. Am J Obstet Gynecol 194: 704-710, 2006.

8. Wu Y, Zhao J, Hu J, Wu XW and Zhu LR: Significance of p16/Ki-67 double immunocytochemical staining in cervical cytology ASCUS, LSIL and ASC-H. Zhonghua Fu Chan Ke Za Zhi 52: 734-739, 2017 (In Chinese; Abstract available in Chinese from the publisher).

9. Kaufmann A, Founta C, Papagiannakis E, Naik P and Fisher A: Standardized digital colposcopy with dynamic spectral imaging for conservative patient management. Case Rep Obstet Gynecol 2017: 5269279, 2017.

10. Louwers J, Zaal A, Kocken M, Ter Harmsel W, Graziosi G Spruijt J, Berkhof J, Balas C, Papagiannakis E, Snijders P, et al: Dynamic spectral imaging colposcopy: Higher sensitivity for detection of premalignant cervical lesions. BJOG 118: 309-318, 2011.

11. Soutter WP, Diakomanolis E, Lyons D, Ghaem-Maghami S, Ajala T, Haidopoulos D, Doumplis D, Kalpaktsoglou C, Sakellaropoulos G, Soliman S, et al: Dynamic spectral imaging: Improving colposcopy. Clin Cancer Res 15: 1814-1820, 2009.

12. Saslow D, Solomon D, Lawson HW, Killackey M, Kulasingam SL, Cain J, Garcia FA, Moriarty AT, Waxman AG, Wilbur DC, et al: American cancer society, American Society for colposcopy and cervical pathology, and American society for clinical pathology screening guidelines for the prevention and early detection of cervical cancer. CA Cancer J Clin 62: 147-172, 2012.

13. Santesso N, Schunemann H, Blumenthal P, De Vuyst H, Gage J, Garcia F, Jeronimo J, Lu R, Luciani S, Quek SC, et al: World Health Organization Guidelines: Use of cryotherapy for cervical intraepithelial neoplasia. Int J Gynaecol Obstet 118: 97-102, 2012.

14. Kelly RS, Patnick J, Kitchener HC and Moss SM; NHSCSP HPV Special Interest Group: HPV testing as a triage for borderline or mild dyskaryosis on cervical cytology: Results from the sentinel sites study. Br J Cancer 105: 983-988, 2011.

15. Li W, Venkataraman S, Gustafsson U, Oyama JC, Ferris DG and Lieberman RW: Using acetowhite opacity index for detecting cervical intraepithelial neoplasia. J Biomed Opt 14: 014020, 2009.

16. Snijders PJ, van den Brule AJ, Jacobs MV, Pol RP and Mejier CJ: HPV DNA detection and typing in cervical scrapes. Methods Mol Med 119: 101-114, 2005.

17. van den Brule AJ, Pol R, Fransen-Daalmeijer N, Schouls LM, Meijer CJ and Snijders PJ: GP5+/6+ PCR followed by reverse line blot analysis enables rapid and high-through identification of human papillomavirus genotypes. J Clin Microbiol 40: 779-787, 2002

18. Rijkaart DC, Berkhof J, van Kemenade FJ, Coupe VM, Hesselink AT, Rozendaal L, Heideman DA, Verheijen RH, Bulk S, Verweij WM, et al: Evaluation of 14 triage strategies for HPV DNA positive women in population based cervical screening. Int J Cancer 130: 602-610, 2012.

19. Pepe M: The statistical evaluation of medical tests for classification and prediction. Oxford: Oxford University Press; 2003.
20. Greiner M: Serodiagnostische tests. Berlin: Springer; 2003.

21. Hanaoka M, Ishikawa T, Ishiquro M, Tokura M, Yamauchi S, Kikuchi A, Uetake H, Yasuno $M$ and Kawano T: Expression of ATF6 as a marker of pre-cancerous atypical change in ulcerative colitis-associated colorectal cancer: A potential role in the management of dysplasia. J Gastroenterol 53:631-641, 2018.

22. Bulkmans NW, Rozendaal L, Snijders PJ, Voorhorst FJ, Boeke AJ, Zandwijken GR, van Kemenade FJ, Verheijen RH, $v$ Groningen K, Boon ME, et al: POBASCAM, a population based randomized controlled trial for implementation of high-risk HPV testing in cervical screening: Design, methods and baseline data of 44, 2012 women. Int J Cancer 110: 94-101, 2004.

23. Zaal A, Louwers JA, Berkhof J, Kocken M, Ter Harmsel WA, Graziosi GC, Spruijt JW, Balas C, Papagiannakis E, Snijders PJ, et al: Agreement between colposcopic impression and histological diagnosis among human papillomavirus type 16 positive women: A clinical trial using dynamic spectral imaging colposcopy. BJOG 119: 537-544, 2012.

24. Ni XG, He S, Xu ZG, Gao L, Lu N, Yuan Z, Lai SQ, Zhang YM, Yi JL, Wang XL, et al: Endoscopic diagnosis of laryngeal cancer and precancerous lesions by narrow band imaging. J Laryngol Otol 125: 288-296, 2011.

25. DeNardis SA, Lavin PT, Livingston J, Salter WR, JamesPatrick N, Papagiannakis E, Olson CG and Weinberg L: Increased detection of precancerous cervical lesions with adjunctive dynamic spectral imaging. Int J Womens Health 9: 717-725, 2017.

26. Belitsos P, Papoutsis D, Rodolakis A, Mesogitis S and Antsaklis A: Three-dimensional power Doppler ultrasound for the study of cervical cancer and precancerous lesions. Ultrasound Obstet Gynecol 40: 576-581, 2012.

27. Moscicki AB, Ma Y, Wibbelsman C, Darragh TM, Powers A, Farhat $\mathrm{S}$ and Shiboski S: Rate of and risks for regression of CIN-2 in adolescents and young women. Obstet Gynecol 116: 1373-1380, 2010

28. Dina R and Argamosa D: The NHS cervical screening programme criteria for evaluating cervical cytology: Comparison of the new with the old. Cytopathology 23: 349-352, 2012.

29. Bekkers RL, van de Nieuwenhof HP, Neesham DE, Hendriks JH, Tan J and Quinn MA: Does experience in colposcopy improve identification of high grade abnormalities. Eur J Obstet Gynecol Reprod Biol 141: 75-78, 2008.

This work is licensed under a Creative Commons Attribution-NonCommercial-NoDerivatives 4.0 International (CC BY-NC-ND 4.0) License. 